\title{
POSITIVE ORIENTED PERIODIC SOLUTIONS OF THE FIRST-ORDER COMPLEX ODE WITH POLYNOMIAL NONLINEAR PART
}

ANDREI BORISOVICH AND WACŁAW MARZANTOWICZ

Received 8 February 2004; Revised 7 March 2004; Accepted 12 March 2004

We study nonlinear ODE problems in the complex Euclidean space, with the right-hand side being polynomial with nonconstant periodic coefficients. As the coefficients functions, we admit only functions with vanishing Fourier coefficients for negative indices. This leads to an existence theorem which relates the number of solutions with the number of zeros of the averaged right-hand side polynomial. A priori estimates of the norms of solutions are based on the Wirtinger-Poincaré-type inequality. The proof of existence theorem is based on the continuation method of Krasnosielski et al., Mawhin et al., and the Leray-Schauder degree. We give a few applications on the complex Riccati equation and some others.

Copyright (c) 2006 Hindawi Publishing Corporation. All rights reserved.

\section{Introduction}

This work is devoted to the $\mathbb{C}$-valued $T$-periodic positive oriented $C^{1}$-smooth solutions of the ordinary differential equation of the form

$$
\dot{u}(t)=\sum_{k=0}^{N} c_{k}(t) u^{k}(t),
$$

where $c_{k}(t), k=0, \ldots, N$, are $\mathbb{C}$-value $T$-periodic positive oriented continuous functions. The study of such solutions was begun in [6]. In this review article we present some new description of our results and their applications to the complex Riccati equation. Some theorems are presented in more general forms and proofs are given in more detail.

The algebra of positive oriented $\mathbb{C}$-valued $T$-periodic functions $C_{+}^{1}(T)$ attracted our attention by two reasons. At first, in $C_{+}^{1}(T)$ we developed a special technique of a priori estimates of solution, which does not occur in the usual algebra $C^{1}(T)$. These estimates give a possibility to separate and to localize the solutions and find favorable conditions for using the degree theory for their determination. 
2 Periodic solutions of the nonlinear complex ODE

In this work we use a priori estimates of solution of two types. At first, the mean value $\mathbf{u}$ of solution $u(t) \in C_{+}^{1}(T)$ is a root of the polynomial equation

$$
0=\sum_{k=0}^{N} \mathbf{c}_{k} \mathbf{u}^{k}
$$

or, shortly $0=P(\mathbf{u})$, where the coefficients of the associated polynomial $P$ are averaging values

$$
\mathbf{c}_{k}=\frac{1}{T} \int_{0}^{T} c_{k}(t) d t, \quad \mathbf{u}=\frac{1}{T} \int_{0}^{T} u(t) d t
$$

and $n=\operatorname{deg} P \leqslant N$. Secondly, some assumptions for the coefficients of (1.1) and for their mean values lead to the following: that the $C^{0}$-norm $\|u\|_{0}$ of solution $u(t) \in C_{+}^{1}(T)$ belongs to the sum of two intervals $\left[r_{0}, r_{1}\right] \cup\left[r_{2},+\infty\right)$, where $0 \leqslant r_{0}<r_{1}<r_{2}$. This estimate is fulfilled only in the algebra of positive oriented functions and is based on the Wirtinger and Poincare inequality (see [19] for details). We study only the first part of set of solutions (with norms in $\left[r_{0}, r_{1}\right]$ ) and prove that it is a compact subset.

In Section 2 we give the definition of the Banach algebra $C_{+}^{k}(T)$ for integer $k \geqslant 0$ and the definition of the associated polynomial $P(z)$. Next, we formulate main Theorem 2.4. This theorem gives a sufficient condition for the existence of a $T$-periodic solution $u(t)$ with $\mathbf{u}=z_{0}$, where $z_{0}$ is a root of $P(z)$. In Section 3 we give all properties of positive oriented functions which we need for study of (1.1). Section 4 is devoted to the proof of a priori estimates.

Section 5 contains the proof of main theorem on the base of the continuation method of Krasnosielski et al. and Mawhin et al. and the Leray-Schauder degree. In some sense our hypothesis is equivalent to an assumption that (1.1) is a small perturbation of (1.2) if the frequency is large enough, which resembles previous approaches to the problem $[15,17,21]$. However, we must say that as well our analytical framework as the required a priori bound have very natural form and lead to a multiplicity result to an effective estimate of the period.

In last Section 6 we give a few applications of our main theorem to the complex Riccati equation and make a comparison with other results in this direction (Lloyd [14], Hassan [12], Campos and Ortega [10], Miklaszewski [20], and Żołądek [22]). We also study the positive oriented periodic solutions on the base of the Nielsen fixed-point theory, see $[1,2,7,8,11]$ and $[3-5]$. In particular, we use the theorems on multiplicity of distinct roots of a complex polynomial [4, 7].

In the end, we remark that the positive oriented solutions of the equation

$$
\dot{u}(t)=f(t, u)
$$

with analytical right part were studied by Marzantowicz in [16]. 


\section{Main theorem}

In this work we look for the solutions $u(t) \in C_{+}^{1}(T)$ of nonlinear differential equation

$$
\dot{u}(t)=\sum_{k=0}^{N} c_{k}(t) u^{k}(t)
$$

with $c_{N}(t) \not \equiv 0$, integer $N \geqslant 2$ and coefficients $c_{k}(t) \in C_{+}^{0}(T)$ for all $k=0,1, \ldots, N$.

Let $C^{p}(T)$ for integer $p \geqslant 0$ and real $T>0$ denotes the Banach algebra of the $C^{p_{-}}$ smooth $\mathbb{C}$-valued $T$-periodic functions $u(t)$ with the norm

$$
\|u\|_{p}=\sum_{k=0}^{p}\left\|u^{(k)}\right\|_{0}, \quad\left\|u^{(k)}\right\|_{0}=\max _{t \in[0, T]}\left|u^{(k)}(t)\right| .
$$

We assign to a given function $u(t) \in C^{0}(T)$ its Fourier series

$$
u(t) \sim \sum_{k=-\infty}^{+\infty} f_{k}(u) \mathbf{e}^{i v k t}, \quad v=\frac{2 \pi}{T}
$$

with the coefficients

$$
f_{k}(u)=\frac{1}{T} \int_{0}^{T} u(t) \mathbf{e}^{-i v k t} d t
$$

Definition 2.1. Let $C_{+}^{p}(T)$ be a set of all functions $u(t) \in C^{P}(T)$ satisfying $f_{k}(u)=0$ for all integer $k<0$. The subset $C_{+}^{p}(T)$ is a Banach subalgebra in $C^{p}(T)$. We call it the algebra of positive oriented functions.

By $\mathbf{A}: C^{0}(T) \rightarrow \mathbb{C}$ we denote the functional of averaging, that is, it assigns to a given function $u(t)$ its mean value

$$
\mathbf{A}(u)=\frac{1}{T} \int_{0}^{T} u(t) d t
$$

Remark that the mean value $\mathbf{A}(u)$ is equal to the 0th Fourier coefficient $f_{0}(u)$.

Definition 2.2. A polynomial $P(z) \in \mathbb{C}[z]$ is associated with (2.1) if

$$
P(z)=\sum_{k=0}^{N} \mathbf{c}_{k} z^{k}
$$

where $\mathbf{c}_{k}=\mathbf{A}\left(c_{k}\right)$ are mean values of $c_{k}(t)$. 
4 Periodic solutions of the nonlinear complex ODE

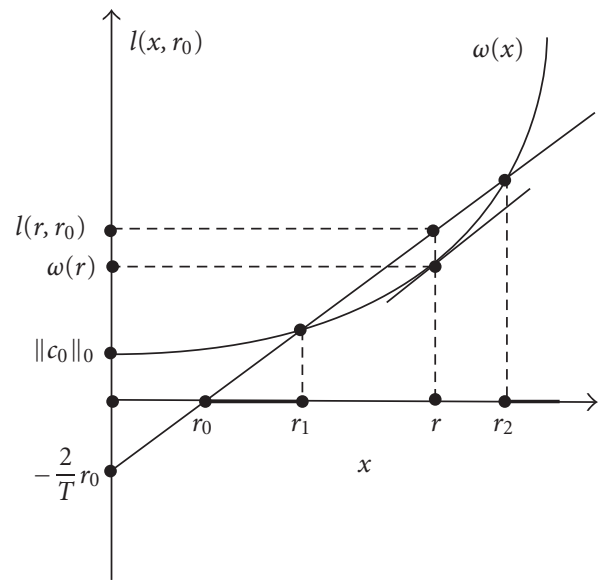

(a)

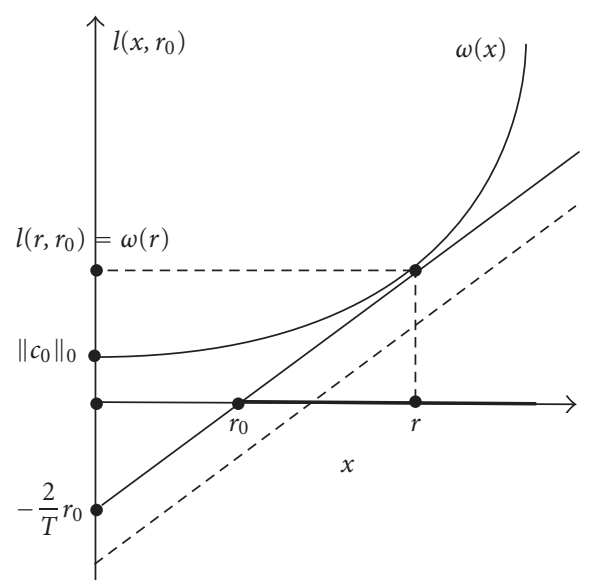

(b)

Figure 2.1

Remark 2.3. If the mean value $\mathbf{c}_{n} \neq 0$ for a certain integer $1 \leqslant n \leqslant N$ and $\mathbf{c}_{k}=0$ for all integer $k=n+1, \ldots, N$, then $\operatorname{deg} P=n$. The degree of associated polynomial $P(z)$ is a less or equal than the degree of right part of (2.1).

We assign also to (2.1) two functions $l, \omega: \mathbb{R}_{+} \rightarrow \mathbb{R}$ defined by formulae

$$
l\left(x, r_{0}\right)=\frac{2}{T}\left(x-r_{0}\right), \quad \omega(x)=\sum_{k=0}^{N}\left\|c_{k}(t)\right\|_{0} x^{k}
$$

depending from real variable $x \geqslant 0$. Function $l\left(x, r_{0}\right)$ is depending on parameter $r_{0} \geqslant 0$ in addition. The graph of $\omega(x)$ starts from the point $\omega(0)=\left\|c_{0}(t)\right\|_{0}$, it is a convex and increasing. The graph of $l\left(x, r_{0}\right)$ is a direct line which starting from the point $l\left(0, r_{0}\right)=$ $-(2 / T) r_{0}$ with the angle $\varphi$, where $\tan \varphi=2 / T$.

On Figures 2.1(a) and 2.1(b) the possibility intersections of the graphs of $l\left(x, r_{0}\right)$ and $\omega(x)$ are showed.

We are now in position to formulate the main theorem.

Theorem 2.4. Consider (2.1). Suppose that

(i) real $T>0$, integer $N \geqslant 2$ and $c_{N}(t) \neq 0$;

(ii) coefficients $c_{k}(t) \in C_{+}^{0}(T)$ for all $k=0,1, \ldots, N$;

(iii) mean value $\mathbf{c}_{n} \neq 0$ for a certain integer $1 \leqslant n \leqslant N$;

(iv) number $z_{0} \in \mathbb{C}$ is a root of the associated polynomial $P(z)$;

(v) equation

$$
\omega(x)=l\left(x, r_{0}\right),
$$




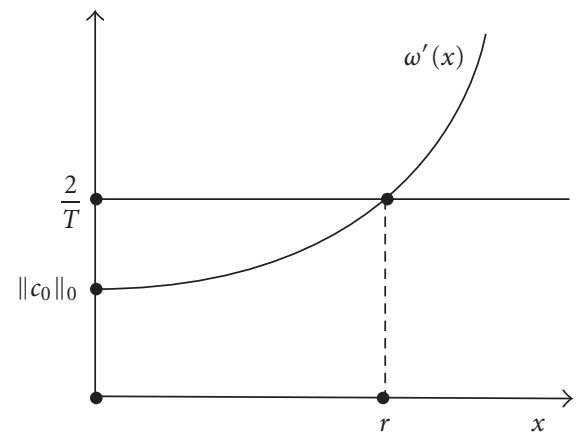

Figure 2.2

where $r_{0}=\left|z_{0}\right|$, has a two distinct roots $r_{1}<r_{2}$ in the interval $[0,+\infty)$, see Figure $2.1(a)$. Then (2.1) has at least one solution $u(t) \in C_{+}^{1}(T)$ with the mean value

$$
\mathbf{A}(u)=z_{0}
$$

Moreover, its $C^{1}$-norm is bounded as follows

$$
r_{0} \leqslant\|u(t)\|_{0} \leqslant r_{1}, \quad\|\dot{u}(t)\|_{0} \leqslant \frac{2}{T}\left(r_{1}-r_{0}\right)
$$

The proof of Theorem 2.4 will be given in Section 5 .

Next, we will study the equation

$$
\omega(x)=l\left(x, r_{0}\right)
$$

Note $r>0$ a point, where the tangent line of $\omega(x)$ is a parallel to $l\left(x, r_{0}\right)$. The point $r$ is not depending from the parameter $r_{0}$ and may be found from the equality $\omega^{\prime}(r)=\tan \varphi$, that is

$$
\sum_{k=1}^{N} k\left\|c_{k}(t)\right\|_{0} r^{k-1}=\frac{2}{T} .
$$

The graph of $\omega^{\prime}(x)$ starts from the points $\omega^{\prime}(0)=\left\|c_{1}(t)\right\|_{0}$ and increases. The condition

$$
\left\|c_{1}(t)\right\|_{0}<\frac{2}{T}
$$

is a necessary and sufficient for an existence of the unique positive solution $r>0$ of the equation $\omega^{\prime}(x)=2 / T$, see Figure 2.2. 
6 Periodic solutions of the nonlinear complex ODE

If for a certain $r_{0} \geqslant 0$ the graphs of $\omega(x)$ and $l\left(x, r_{0}\right)$ have a two distinct intersection points $r_{1}<r_{2}$ in the interval $[0,+\infty)$, then $r_{1}<r<r_{2}$ and $\omega(r)<l\left(r, r_{0}\right)$, that is

$$
\sum_{k=0}^{N}\left\|c_{k}(t)\right\|_{0} r^{k}<\frac{2}{T}\left(r-r_{0}\right)
$$

see Figure 2.1(a). Moreover:

Property 2.5. The fulfilment of the inequalities (2.13) and (2.14) for a certain $r_{0} \geqslant 0$ is a necessary and sufficient condition for an existence in the interval $[0,+\infty)$ of the two distinct points of intersection of the graphs of $\omega(x)$ and $l\left(x, r_{0}\right)$.

Remark 2.6. The inequality (2.14) may be written in the form

$$
r_{0}<r-\frac{T}{2} \sum_{k=0}^{N}\left\|c_{k}(t)\right\|_{0} r^{k}
$$

what is a very convenient for the application to the differential equation (2.1) because the constant $r_{0}=\left|z_{0}\right|$ in the left part of (2.15) depending only from the mean values $\mathbf{c}_{k}$ of the coefficients of (2.1) and the constant in the right part of the inequality (2.15) depending from its $C^{0}$-norms and period $T$.

\section{Algebra of periodic $C_{+}^{p}$-functions}

At first we study the properties of the Banach algebras $C_{+}^{p}(T)$, integer $p \geqslant 0$ and $T>0$, which are necessary for the proof of main Theorem 2.4.

In $C^{0}(T)$ consider the following linear subspace of the trigonometrical polynomials

$$
E(T)=\left\{c_{0}+\sum_{k=1}^{m} c_{k} \mathbf{e}^{k}(t): c_{0}, c_{k} \in \mathbb{C}, m=1,2, \ldots\right\}
$$

where

$$
\mathbf{e}(t)=\mathbf{e}^{i v t}, \quad v=\frac{2 \pi}{T}
$$

It is clear, that $E(T)$ is a subspace of every $C^{P}(T)$.

Definition 3.1. We will denote by $C_{+}^{p}(T)$ the closure of subspace $E(T)$ in $C^{p}(T)$ with respect to its norm $\|\cdot\|_{p}$.

Definition 3.1 is equivalent to Definition 2.1, see [6].

We assign to a given period $T>0$ the two dimensional open disc on the complex plane

$$
D_{\tau}=\{z:|z|<\tau\}, \quad \tau=\frac{T}{2 \pi} .
$$


Let us denote by $S_{\tau}$ its boundary and by $\bar{D}_{\tau}$ the closed disc. Remark that the length of $S_{\tau}$ is equal to $T$. For a given $\mathbb{C}$-valued function $g(z)$, which is defined in some open neighborhood of $\bar{D}_{\tau}$, we assign its restriction $u(t)$ to the boundary $S_{\tau}$ by following formula

$$
u(t)=g\left(\tau \mathbf{e}^{i v t}\right)
$$

Remark that the each trigonometrical polynomial

$$
v(t)=\sum_{k=0}^{m} c_{k} \mathbf{e}^{i v k t}
$$

is a restriction to the circle $S_{\tau}$ of the corresponding holomorphic function

$$
h(z)=\sum_{k=0}^{m}\left(c_{k} \nu^{k}\right) z^{k} .
$$

Let $H\left(\bar{D}_{\tau}\right)$ denotes the linear space of the $\mathbb{C}$-valued functions $h(z)$ defined and holomorphic in some open neighborhood of $\bar{D}_{\tau}$. Let $C^{p}\left(S_{\tau}\right)$ denotes the Banach algebra of the $C^{p}$-smooth $\mathbb{C}$-valued functions $u(t)$ defined on the circle $S_{\tau}$. Note that $C^{p}\left(S_{\tau}\right)$ can be identified with $C^{p}(T)$. We call $H\left(S_{\tau}\right)$ or $H(T)$ the set of restrictions to the boundary $S_{\tau}$ of all functions $h(z) \in H\left(\bar{D}_{\tau}\right)$. The set $H(T)$ is a linear subspace in $C^{p}(T)$ for all integer $p \geqslant 0$.

Definition 3.2. We will denote by $C_{+}^{p}(T)$ the closure of subspace $H(T)$ in $C^{p}(T)$ with respect to its norm $\|\cdot\|_{p}$.

Definition 3.2 is equivalent to Definitions 2.1 and 3.1, see [6].

Property 3.3. The functional of averaging $\mathbf{A}: C_{+}^{p}(T) \rightarrow \mathbb{C}$, defined by (2.2), is a linear and multiplicative, that is, for every $u(t), v(t) \in C_{+}^{p}(T)$ and every $\alpha, \beta \in \mathbb{C}$ we have

$$
\begin{aligned}
\mathbf{A}(\alpha u+\beta v) & =\alpha \mathbf{A}(u)+\beta \mathbf{A}(v), \\
\mathbf{A}(u v) & =\mathbf{A}(u) \mathbf{A}(v) .
\end{aligned}
$$

Property 3.3 may be simple verified for $u(t), v(t) \in E(T)$, see formula (3.1). Property 3.3 for all functions in $C_{+}^{p}(T)$ follows from the continuity of $\mathbf{A}$.

Definition 3.4. We call $V_{+}^{p}(T)$, integer $p \geqslant 0$ and real $T>0$, the kernel subspace of the averaging functional $\mathbf{A}: C_{+}^{p}(T) \rightarrow \mathbb{C}$ defined by (2.3).

Recall that $V_{+}^{p}(T)$ is a set of all functions $v(t) \in C_{+}^{p}(T)$ having $f_{0}(v)=0$, where $f_{0}(v)$ is its 0th Fourier coefficient.

It is well known that the kernel subspace of multiplicative linear functional is a maximal ideal in the Banach algebra. Let $V_{0}$ denotes the subspace of constant complex functions with the based element $e_{0}(t) \equiv 1$. We will write $V_{0} \sim \mathbb{C}$. We will be different: $e_{0}(t) \equiv$ 1 is an element of the Banach algebra $C_{+}^{p}(T)$ and 1 is an element of $\mathbb{C}$. 
8 Periodic solutions of the nonlinear complex ODE

Property 3.5. The Banach algebra $C_{+}^{p}(T)$, integer $p \geqslant 0$ and real $T>0$, may be written as a direct sum

$$
C_{+}^{p}(T)=V_{0} \oplus V_{+}^{p}(T)
$$

The every function $v(t) \in V_{+}^{p}(T)$ may be written as

$$
v(t)=\mathbf{e}^{i v t} u(t)
$$

where $u(t) \in C_{+}^{p}(T)$. Therefore, the Banach algebras $C_{+}^{p}(T)$ and $V_{+}^{p}(T)$ are isometric. For a given integer $m \geqslant 0$ we have a decomposition

$$
C_{+}^{p}(T)=V_{0} \oplus V_{1}(T) \oplus \cdots \oplus V_{m}(T) \oplus V_{+m}^{p}(T),
$$

where

$$
V_{k}(T)=\left\{c \mathrm{e}^{i v k t}: c \in \mathbb{C}\right\}, \quad k=0,1, \ldots, m,
$$

and $V_{+m}^{p}(T)$ is a Banach algebra of all functions $v(t) \in C_{+}^{p}(T)$ satisfying

$$
f_{k}(v)=0, \quad k=0,1, \ldots, m
$$

Note that algebras $V_{+m}^{p}(T)$ in the decompositions (3.10) for all integer $m \geqslant 0$ are isometric to $C_{+}^{p}(T)$. Moreover, $V_{+0}^{p}(T)=C_{+}^{p}(T)$ and $V_{+1}^{p}(T)=V_{+}^{p}(T)$.

Definition 3.6. We call $J$ the projector $J: C_{+}^{p}(T) \rightarrow C_{+}^{p}(T)$ to the subspace $V_{0}$ along the ideal $V_{+}^{p}(T)$ defined by formula

$$
J(u)=\mathbf{A}(u) e_{0}
$$

It is a multiplicative linear bounded map with $\operatorname{Im} J=V_{0}$ and $\operatorname{Ker} J=V_{+}^{p}(T)$ preserving all elements from $V_{0}$.

Definition 3.7. We will denote by $D$ the operator of derivative $D: C_{+}^{p}(T) \rightarrow C_{+}^{p-1}(T)$, integer $p \geqslant 1$ and real $T>0$, defined by the formula

$$
D(u)(t)=\dot{u}(t) .
$$

Property 3.8. The operator $D$ is a linear bounded. It belongs to the class $\Phi_{0}$, that is, is a Fredholm map of index 0. Moreover, it has

$$
\operatorname{Ker} D=V_{0}, \quad \operatorname{Im} D=V_{+}^{p-1}(T) .
$$

The restriction $D: V_{+}^{p}(T) \rightarrow V_{+}^{p-1}(T)$ is an isomorphism. It is naturally, because all components $V_{k}(T), k \in \mathbb{N}$, in the decomposition (3.10) are the eigne-subspaces of the linear map $D$. 
Property 3.9. Let $\mathbf{A}: C_{+}^{0}(T) \rightarrow \mathbb{C}$ denotes the functional of averaging, see (2.2), and $u(t) \in$ $C_{+}^{1}(T)$. Then we have

$$
\mathbf{A}(\dot{u})=0 .
$$

To prove Property 3.9 consider the differential operator $D: C_{+}^{1}(T) \rightarrow C_{+}^{0}(T)$. We have

$$
V_{+}^{0}(T)=\operatorname{Im} D=\operatorname{Ker} \mathbf{A} .
$$

Definition 3.10. We will denote by $L$ the differential operator $L: C_{+}^{p}(T) \rightarrow C_{+}^{p-1}(T)$, integer $p \geqslant 1$ and real $T>0$, defined by the formula

$$
L(u)(t)=\dot{u}(t)+u(t) .
$$

Property 3.11. The operator $L$ is an isomorphism.

To prove Property 3.11 we write the operator $L$ as a sum

$$
L=D+E \circ I,
$$

where $I: C_{+}^{p}(T) \rightarrow C_{+}^{p}(T)$ is the identity map and $E: C_{+}^{p}(T) \rightarrow C_{+}^{p-1}(T)$ is a natural embedding of the Banach algebras. Recall that $E$ is a completely continuous. Following the superposition $E \circ I$ is a completely continuously too. In the sum (3.19) the first operator belongs to the class $\Phi_{0}$ and the second is a completely continuous. Therefore $L \in \Phi_{0}$. It is not difficult to verify that $\operatorname{Ker} L=\{0\}$. Following the map $L$ is an isomorphism.

\section{A priori estimates of solutions in $C_{+}^{p}$-algebra}

The proof of the main Theorem 2.4 is based on the homotopy

$$
\dot{u}(t)=\sum_{k=0}^{N}\left(\lambda c_{k}(t) u^{k}(t)+(1-\lambda) \mathbf{c}_{k} \mathbf{A}(u)^{k}\right)
$$

with parameter $\lambda \in[0,1]$, where the constants $\mathbf{c}_{k}=\mathbf{A}\left(c_{k}\right)$ are mean values of $c_{k}(t), \mathbf{A}(u)$ is a mean value of $u(t)$. Remark that the homotopy (4.1) is thought to deformation (2.1) to a simpler equation $\dot{u}=P(\mathbf{u})$, where $P$ is a polynomial associated with (2.1). The simpler equation has only constant solutions $u(t) \equiv z, \dot{u}(t) \equiv 0$, where $z$ is a root of $P$. In this section we will give some a priori properties of the solutions of $(4.1)$ in $C^{1}(T)$ and $C_{+}^{1}(T)$, which we need to prove the main Theorem 2.4.

We will use the following inequality of the Wirtinger-Poincare type, see [19] and [6] for details.

Property 4.1. Let $u(t) \in C^{1}(T)$ be a $T$-periodic function, $\mathbf{u}=\mathbf{A}(u)$ its mean value and $\|u\|_{0}$ its $C^{0}$-norm, see formulae (2.5) and (2.2). Then

$$
0 \leqslant\|u\|_{0}-|\mathbf{u}| \leqslant \frac{T}{2}\|\dot{u}\|_{0} .
$$


Proof. For every $0 \leqslant t_{0} \leqslant T$ we have

$$
0 \leqslant\|u(t)\|_{0}-\left|u\left(t_{0}\right)\right| \leqslant\left\|u(t)-u\left(t_{0}\right)\right\|_{0} \leqslant \frac{T}{2}\|\dot{u}(t)\|_{0} .
$$

On the other hand for the mean value $\mathbf{u}$ of any periodic function $u(t) \in C^{1}(T)$ we have $|\mathbf{u}| \leqslant\left|u\left(t_{*}\right)\right|$ for some $t_{*} \in[0, T]$. This leads to Property 4.1.

Property 4.2. Consider (4.1) with real $T>0$, integer $N \geqslant 2$ and all coefficient $c_{k}(t) \in$ $C^{0}(T)$. Let $u(t) \in C^{1}(T)$ is a solution for some $\lambda \in[0,1]$. Then the following inequality holds:

$$
\frac{2}{T}\left(\|u\|_{0}-|\mathbf{u}|\right) \leqslant \sum_{k=0}^{N}\left\|c_{k}\right\|_{0}\|u\|_{0}^{k}
$$

or, shortly,

$$
l\left(\|u\|_{0},|\mathbf{u}|\right) \leqslant \omega\left(\|u\|_{0}\right)
$$

Recall that $\mathbf{u}=\mathbf{A}(u)$ is a mean value of $u(t)$ and

$$
\|u\|_{0}=\max _{t \in[0, T]}|u(t)|
$$

Proof. To prove Property 4.2 we calculate the $C^{0}$-norms from the left and right parts of (4.1) and use the inequalities $\left|\mathbf{c}_{k}\right| \leqslant\left\|c_{k}\right\|_{0}$ for all $k=0,1, \ldots, N$ and $|\mathbf{A}(u)| \leqslant\|u\|_{0}$. We get

$$
\|\dot{u}\|_{0} \leqslant \sum_{k=0}^{N}\left\|c_{k}\right\|_{0}\|u\|_{0}^{k}
$$

independently from the parameter $\lambda \in[0,1]$. From (4.2) we get

$$
\frac{2}{T}\left(\|u\|_{0}-|\mathbf{u}|\right) \leqslant\|\dot{u}\|_{0}
$$

The inequalities (4.7) and (4.8) lead Property 4.2.

Property 4.3. Let $u(t) \in C_{+}^{1}(T)$ is a solution of (4.1) for some $\lambda \in[0,1]$, where integer $N \geqslant 2$ and all coefficients $c_{k}(t) \in C_{+}^{0}(T)$. Then its mean value $\mathbf{u}=\mathbf{A}(u)$ is a root of the associated polynomial $P(z)$, that is

$$
0=\sum_{k=0}^{n} \mathbf{c}_{k} \mathbf{u}^{k}
$$

To prove Property 4.3 we calculate the mean values from the left and right parts of (4.1) and apply Properties 3.3 and 3.9.

Remark that $(4.1)$ for each $\lambda \in[0,1]$ has a same associated polynomial $P(z)$ at degree $n \leqslant N$. Let $P^{-1}(0)=\left\{z_{1}, \ldots, z_{m}\right\}$, where integer $1 \leqslant m \leqslant n$. For a given root $z_{j}$ we assign 
the fiber $F\left(z_{j}\right)$ in the space $C_{+}^{1}(T)$ defined by formula

$$
F\left(z_{j}\right)=\left\{u(t): \mathbf{A}(u)=z_{j}\right\}, \quad j=1, \ldots, m .
$$

Note that all solutions of $(4.1)$ in the algebra $C_{+}^{1}(T)$ belong to the set $F$, which is a sum of all fibers

$$
F=F\left(z_{1}\right) \cup \cdots \cup F\left(z_{m}\right) .
$$

Theorem 4.4. Consider the family (4.1) with parameter $\lambda \in[0,1]$. Suppose that

(i) real $T>0$, integer $N \geqslant 2$ and $c_{N}(t) \neq 0$;

(ii) coefficients $c_{k}(t) \in C_{+}^{0}(T)$ for all $k=0,1, \ldots, N$;

(iii) mean value $\mathbf{c}_{n} \neq 0$ for a certain integer $1 \leqslant n \leqslant N$ and $\mathbf{c}_{k}=0$ for all $k>n$;

(iv) number $z_{0} \in \mathbb{C}$ is a root of the associated polynomial $P(z)$;

(v) function $u(t) \in C_{+}^{1}(T)$ is a solution of (4.1) for some $\lambda \in[0,1]$ and $\mathbf{A}(u)=z_{0}$. Then, its $C^{0}$-norm and mean value satisfy a priori inequality

$$
l\left(\|u\|_{0},\left|z_{0}\right|\right) \leqslant \omega\left(\|u\|_{0}\right)
$$

where the functions $l\left(x, r_{0}\right)$ and $\omega(x)$ defined by formulae (2.7).

Theorem 4.4 follows directly from Properties 4.2 and 4.3.

Remark 4.5. If the supposition (v) of the main Theorem 2.4 is fulfilled, see Figure 2.1(a) and Property 2.5, then for each solution $u(t) \in F\left(z_{0}\right) \subset C_{+}^{1}(T)$ of (2.1) its $C^{0}$-norm $\|u(t)\|_{0}$ belongs to the one of two intervals $\left[r_{0}, r_{1}\right]$ or $\left[r_{2},+\infty\right)$, where $r_{0}=\left|z_{0}\right|$ and $r_{1}<r_{2}$ are positive roots of the equation $l\left(x, r_{0}\right)=\omega(x)$. If the supposition (v) of the main Theorem 2.4 is not fulfilled, then $\|u(t)\|_{0}$ belongs to $\left[r_{0},+\infty\right)$, see Figure 2.1(b).

\section{Proof of main theorem}

We are now in position to prove the main Theorem 2.4. Introduce the following operators acted in the Banach algebras of positive oriented functions:

$$
L: C_{+}^{1}(T) \longrightarrow C_{+}^{0}(T), \quad L u(t)=\dot{u}(t)+u(t),
$$

is an isomorphism $L=D+I$, see Property 3.11;

$$
Q: C_{+}^{0}(T) \longrightarrow C_{+}^{0}(T), \quad Q u(t)=\sum_{k=0}^{N} c_{k}(t) u^{k}(t)+u(t)
$$

is a nonlinear continuous map (evaluation Niemytskij operator);

$$
E: C_{+}^{1}(T) \longrightarrow C_{+}^{0}(T), \quad E u(t)=u(t),
$$


is a natural embedding of the Banach algebras (completely continuous map);

$$
J: C_{+}^{1}(T) \longrightarrow C_{+}^{1}(T), \quad J u(t)=\mathbf{A}(u) e_{0}(t),
$$

is a projector to the subspace $V_{0} \sim \mathbb{C}$, where $e_{0}(t) \equiv 1$ is an element of $C_{+}^{1}(T)$.

Reduce the differential equation (2.1) to a fixed point problem $u=G(u)$ with the completely continuous map $G: C_{+}^{1}(T) \rightarrow C_{+}^{1}(T)$. Add the function $u(t)$ to the left and right parts of (2.1). We get

$$
\dot{u}(t)+u(t)=\sum_{k=0}^{N} c_{k}(t) u^{k}(t)+u(t) .
$$

Equation (5.5) may be written in the operator form

$$
L(u)=Q \circ E(u)
$$

where the operators $L, Q, E$ are defined by (5.1), (5.2), and (5.3). Equation (5.6) may be written in the form

$$
u=L^{-1} \circ Q \circ E(u)
$$

with the help of the invertibility of $L$, see Property 3.11. Denote

$$
G=L^{-1} \circ P \circ E
$$

Then, the operator equation (5.7) or differential equation (2.1) may be written as a fixed points problem $u=G(u)$ for the completely continuous map $G: C_{+}^{1}(T) \rightarrow C_{+}^{1}(T)$ defined by formula (5.8). The map $G$ is a completely continuous because one of the elements of decomposition (5.8) is a completely continuous embedding of the Banach algebras, see (5.3).

The actions of all operators are illustrated on the following scheme

$$
C_{+}^{1}(T) \stackrel{L}{\longrightarrow} C_{+}^{0}(T) \stackrel{Q}{\longleftarrow} C_{+}^{0}(T) \stackrel{E}{\longleftarrow} C_{+}^{1}(T) .
$$

Recall that the proof of the main Theorem 2.4 is based on the homotopy (4.1), which is thought to deform (2.1) to a simpler equation. Write all of this equations together:

$$
\begin{array}{ll}
\dot{u}(t)=\sum_{k=0}^{N} c_{k}(t) u^{k}(t), & \lambda=1, \\
\dot{u}(t)=\sum_{k=0}^{N}\left[\lambda c_{k}(t) u^{k}(t)+(1-\lambda) \mathbf{c}_{k} \mathbf{A}(u)^{k}\right], & 0 \leqslant \lambda \leqslant 1, \\
\dot{u}(t)=\sum_{k=0}^{N} \mathbf{c}_{k} \mathbf{A}(u)^{k}, & \lambda=0 .
\end{array}
$$


Write this homotopy in the operator form:

$$
\begin{array}{ll}
u=G(u), & \lambda=1, \\
u=G_{\lambda}(u), & 0 \leqslant \lambda \leqslant 1, \\
u=J \circ G(u), & \lambda=0 .
\end{array}
$$

Recall, see Property 3.5, that the Banach algebra $C_{+}^{1}(T)$ may be written as a direct sum

$$
C_{+}^{1}(T)=V_{+}^{1}(T) \oplus V_{0}
$$

with the finite-dimensional subspace

$$
V_{0}=\left\{C e_{0}(t): C \in \mathbb{C}\right\} \sim \mathbb{C},
$$

where $e_{0}(t) \equiv 1$ is an element of $C_{+}^{1}(T)$. The map $J: C_{+}^{1}(T) \rightarrow C_{+}^{1}(T)$ is a projector to $V_{0}$ along $V_{+}^{1}(T)$, see Definition 3.6.

Following the operator $J \circ G: C_{+}^{1}(T) \rightarrow C_{+}^{1}(T)$ acts by right

$$
J \circ G(u)(t)=\sum_{k=0}^{N}\left[\mathbf{c}_{k} \mathbf{A}(u)^{k}+\mathbf{A}(u)\right] e_{0}(t)
$$

and it has a finite-dimensional image, see Figure 5.1. All its fixed points belong to the finite-dimensional subspace, that is

$$
\operatorname{Im}(J \circ G) \subset V_{0}, \quad \operatorname{Fix}(J \circ G) \subset V_{0} .
$$

From Definition 2.2 of the associated polynomial $P(z)$, Property 4.3 and the equality (5.14) we get

$$
\left.(J \circ G-I)\right|_{V_{0} \sim \mathbb{C}}=P,
$$

where the left part of (5.16) is a restriction of the map $J \circ G-I$ to the finite-dimensional subspace $V_{0}$. On the base of (5.16) we get

$$
\operatorname{Fix}(J \circ G)=P^{-1}(0)
$$

or, more exactly, the equation

$$
u=J \circ G(u)
$$

is equivalent to the system

$$
\begin{gathered}
u(t) \equiv z, \quad z \in \mathbb{C}, \\
P(z)=0, \quad P: \mathbb{C} \longrightarrow \mathbb{C} .
\end{gathered}
$$

Property 5.1. Consider the family of (5.11) with parameter $\lambda \in[0,1]$. Let the number $z_{0} \in \mathbb{C}$ is a root of the associated polynomial $P(z)$. Then the simpler equation $u=J \circ G(u)$ for $\lambda=0$ has a solution $u_{0}(t) \equiv z_{0}$. 
14 Periodic solutions of the nonlinear complex ODE

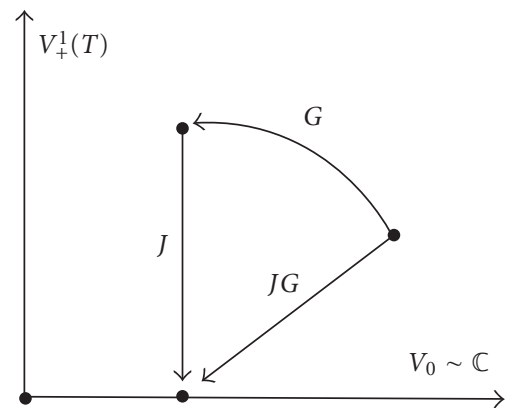

Figure 5.1

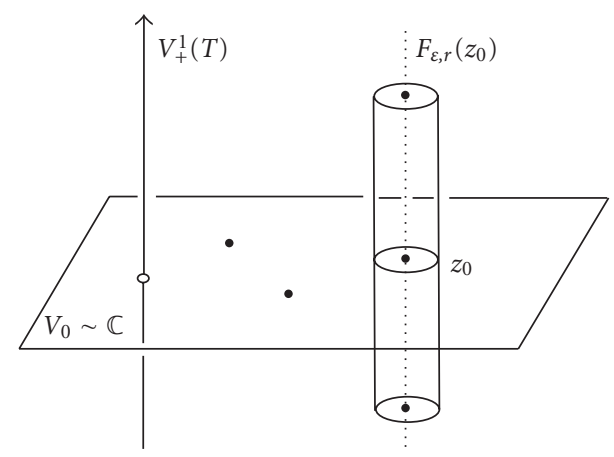

Figure 5.2

Recall, see Property 4.3 and Figure 5.2, that this solution belongs to the fiber

$$
F\left(z_{0}\right)=\left\{u(t): \mathbf{A}(u)=z_{0}\right\}
$$

We would like to show on the base of the Leray-Schauder degree that the root of $u=G_{\lambda}(u)$ will be preserved in the fiber $F\left(z_{0}\right)$ for all $\lambda \in[0,1]$.

Now, we are in position to apply the Leray-Schauder degree.

Lemma 5.2. Let $U$ is a bounded open connected set in $C_{+}^{1}(T)$. Consider the intersection

$$
U_{\mathbb{C}}=U \cap V_{0} .
$$

Assume that $U_{\mathbb{C}} \neq \varnothing$, then it is a bounded open connected set in $V_{0} \sim \mathbb{C}$. Suppose that the boundary $\partial U_{\mathbb{C}}$ there is not a roots of the associated polynomial $P(z)$. Then,

$$
\operatorname{deg}(J \circ G-I, U, 0)=\operatorname{deg}\left(P, U_{\mathbb{C}}, 0\right),
$$

where deg denotes the Leray-Schauder degree. 
Choose the sufficiently small open neighborhood of $z_{0}$ in $\mathbb{C}$

$$
\mathbb{C}_{\varepsilon}\left(z_{0}\right)=\left\{z:\left|z-z_{0}\right|<\varepsilon\right\},
$$

such that its closure does not consist others roots of the associated polynomial $P(z)$.

Choose also the real number $r>0$ such that $r_{1}<r<r_{2}$, see supposition (v) of Theorem 2.4 and Figure 2.1(a). For example, $r$ may be chosen as a unique root of (2.12).

In Banach algebra $C_{+}^{1}(T)$ define the open bounded connected set by following

$$
F_{\varepsilon, r}\left(z_{0}\right)=\left\{u(t):\left|\mathbf{A}(u)-z_{0}\right|<\varepsilon,\|u\|_{0}<r,\|\dot{u}\|_{0}<\sum_{k=0}^{N}\left\|c_{k}\right\|_{0} r^{k}\right\},
$$

see Figure 5.2. Remark that

$$
F_{\varepsilon, r}\left(z_{0}\right) \cap V_{0}=\mathbb{C}_{\varepsilon}\left(z_{0}\right) .
$$

For each solution $u(t) \in F\left(z_{0}\right) \subset C_{+}^{1}(T)$ of the equation $u=G_{\lambda}(u)$ for some $\lambda \in[0,1]$ its $C^{0}$-norm $\|u(t)\|_{0}$ belongs to the one of two intervals $\left[r_{0}, r_{1}\right]$ or $\left[r_{2},+\infty\right)$. It follows from Theorem 4.4 and supposition (v) of Theorem 2.4. We get

$$
\|u\|_{0} \leqslant r_{1}<r \quad \text { or } \quad r<r_{2} \leqslant\|u\|_{0} .
$$

From (5.26) and Property 4.3 we get

$$
\operatorname{Fix} G_{\lambda} \cap \partial F_{\varepsilon, r}\left(z_{0}\right)=\varnothing
$$

for all $\lambda \in[0,1]$.

Finally. Let $z_{0} \in \mathbb{C}$ is a root of the associated polynomial $P(z)$. Then:

$$
\begin{gathered}
\operatorname{deg}\left(P, \mathbb{C}_{\varepsilon}\left(z_{0}\right), 0\right) \neq 0 ; \\
u_{0}(t) \equiv z_{0} \text { is a solution of } u=J \circ G(u) ; \\
u_{0}(t) \text { belongs to the set } F_{\varepsilon, r}\left(z_{0}\right) .
\end{gathered}
$$

Using the Leray-Schauder degree and Lemma 5.2, we get

$$
\begin{aligned}
\operatorname{deg}\left(I-G, F_{\varepsilon, r}\left(z_{0}\right), 0\right) & =\operatorname{deg}\left(I-G_{\lambda}, F_{\varepsilon, r}\left(z_{0}\right), 0\right) \\
& =\operatorname{deg}\left(I-J \circ G, F_{\varepsilon, r}\left(z_{0}\right), 0\right) \\
& =\operatorname{deg}\left(-P, \mathbb{C}_{\varepsilon}\left(z_{0}\right), 0\right) \neq 0 .
\end{aligned}
$$

Following the equation $u=G(u)$ has at least one solution $u(t) \in F_{\varepsilon, r}\left(z_{0}\right) \subset C_{+}^{1}(T)$.

\section{Applications}

In this section we first give some modifications of the main Theorem 2.4, which are convenient to the applications to particular differential equations. Now, we need a following simple result from classical algebra. 
Property 6.1. On the complex plane consider the following polynomial equation

$$
0=\sum_{k=0}^{n} \mathbf{c}_{k} z^{k}
$$

where all coefficient $\mathbf{c}_{k} \in \mathbb{C}$, integer $0 \leqslant k \leqslant n$, and $\mathbf{c}_{n} \neq 0$. Denote

$$
\mathbf{m}=\frac{1}{\left|\mathbf{c}_{n}\right|} \sum_{k=0}^{n-1}\left|\mathbf{c}_{k}\right|
$$

Then, each root $z_{0}$ of (6.1) is satisfied to one of two following a priory estimates

$$
\left|z_{0}\right| \leqslant \mathbf{m}^{1 / n}, \quad \text { if } \mathbf{m}<1
$$

or

$$
\left|z_{0}\right| \leqslant \mathbf{m}, \quad \text { if } \mathbf{m} \geqslant 1 .
$$

From Theorem 2.4, Property 2.5, Remark 2.6, and Property 6.1 we get following theorem.

Theorem 6.2. Consider the differential equation (2.1). Suppose that

(i) real $T>0$, integer $N \geqslant 2$ and $c_{N}(t) \neq 0$;

(ii) coefficients $c_{k}(t) \in C_{+}^{0}(T)$ for all $k=0,1, \ldots, N$;

(iii) $C^{0}$-norm $\left\|c_{1}(t)\right\|_{0}<2 / T$;

(iv) mean value $\mathbf{c}_{n} \neq 0$ for a certain integer $1 \leqslant n \leqslant N$ and $\mathbf{c}_{k}=0$ for $n<k \leqslant N$;

(v) one of the two following inequalities holds

$$
\mathbf{m}^{1 / n}<r-\frac{T}{2} \sum_{k=0}^{N}\left\|c_{k}(t)\right\|_{0} r^{k}, \quad \text { if } \mathbf{m}<1,
$$

or

$$
\mathbf{m}<r-\frac{T}{2} \sum_{k=0}^{N}\left\|c_{k}(t)\right\|_{0} r^{k}, \quad \text { if } \mathbf{m} \geqslant 1,
$$

where $r>0$ is a unique root of (2.12) and $\mathbf{m} \geqslant 0$ is defined by (6.2). Then, for each root $z_{0}$ of the associated polynomial $P(z)$ correspond at least one solution $u(t) \in C_{+}^{1}(T)$ of the differential equation (2.1) with the mean value $\mathbf{A}(u)=z_{0}$ and $C^{0}$-norm less than $r$.

The proof of Theorem 6.2 is based on the fact, that the inequality (6.5) or (6.6) leads the inequality (2.14) or (2.15) for all roots of associated polynomial $P(z)$ simultaneously. Remark 6.3. The supposition (iv) implies that $\operatorname{deg} P=n$.

We will give a sufficient condition for the existence in $C_{+}^{1}(T)$ of $n$ distinct solutions of (2.1). It states that if associated polynomial $P(z)$ has $n$ distinct roots in $\mathbb{C}$. 
Theorem 6.4. Consider the differential equation (2.1). Let supposition (i)-(v) of Theorem 6.2 are fulfilled. Assume that all coefficients $\mathbf{c}_{k}, 0 \leqslant k \leqslant n$, satisfy the conditions

$$
\begin{gathered}
\left|\mathbf{c}_{0}\right|>\left|\mathbf{c}_{1}\right|+\left|\mathbf{c}_{2}\right|+\cdots+\left|\mathbf{c}_{s}\right| \\
\left|\mathbf{c}_{s+1}\right|>\left|\mathbf{c}_{0}\right|+\cdots+\left|\mathbf{c}_{s}\right|+\left|\mathbf{c}_{s+2}\right|+\cdots+\left|\mathbf{c}_{n}\right|
\end{gathered}
$$

for a certain integer $1 \leqslant s \leqslant n$. Then, the differential equation (2.1) has at least $s$ distinct solutions in the algebra $C_{+}^{1}(T)$ with $C^{0}$-norm less than $r$.

The statement follows from Theorem 6.2 and example to corollary and proposition 3 of [8]. The fulfilment of (6.7) is a sufficient condition for the existence of $s$ distinct roots of the associated polynomial $P(z)$. This fact was proved in [8] on the base of the Nielsen Fixed Point Theory, see also [7] and [11].

Property 6.5. On the complex plane consider the polynomial equation

$$
0=z^{n}+\sum_{k=0}^{n-1} \mathbf{c}_{k} z^{k}
$$

where all coefficient $\mathbf{c}_{k} \in \mathbb{C}$, integer $0 \leqslant k \leqslant n-1$. If

$$
\left|1+\mathbf{c}_{0}\right|+\sum_{k=1}^{n-1}\left|\mathbf{c}_{k}\right|<\frac{1}{2}
$$

Then, (6.8) has $n$ distinct roots $z_{1}, \ldots, z_{n}$ such that

$$
\left(\frac{1}{2}\right)^{1 / n} \leqslant\left|z_{j}\right| \leqslant 1+\frac{1}{2}, \quad j=1, \ldots, n .
$$

Property 6.5 follows from Theorem 2.1 in [4], which was proved on the base of Nielsen Fixed Point Theory (see also $[3,5]$ ).

From Theorem 6.2 and Property 6.5 we get the following theorem.

Theorem 6.6. Consider the differential equation (2.1). Suppose that

(i) real $T>0$, integer $N \geqslant 2$ and $c_{N}(t) \neq \equiv$;

(ii) coefficients $c_{k}(t) \in C_{+}^{0}(T)$ for all $k=0,1, \ldots, N$;

(iii) $C^{0}$-norm $\left\|c_{1}(t)\right\|_{0}<2 / T$ and $r>0$ is a unique root of (2.12);

(iv) following inequality holds

$$
\frac{3}{2}<r-\frac{T}{2} \sum_{k=0}^{N}\left\|c_{k}(t)\right\|_{0} r^{k}
$$

(v) mean value $\mathbf{c}_{n}=1$ for a certain integer $1 \leqslant n \leqslant N$ and $\mathbf{c}_{k}=0$ for $n<k \leqslant N$;

(vi) following inequality holds

$$
\left|1+\mathbf{c}_{0}\right|+\sum_{k=1}^{n-1}\left|\mathbf{c}_{k}\right|<\frac{1}{2} .
$$


Then, (2.1) has at least $n$ distinct solutions in the Banach algebra $C_{+}^{1}(T)$ with $C^{0}$-norm less than $r$.

Theorem 6.6 is a very convenient for applications because the verification of assumptions (iii) and (iv), which connected with $C^{0}$-norm of coefficients and period $T$, is separated from the verification of assumptions (v) and (vi), which connected with its mean values. Theorem 6.6 may be applied to the differential equations in the form

$$
\dot{u}(t)=u^{n}(t)+\sum_{k=0}^{n-1} c_{k}(t) u^{k}(t),
$$

where $c_{n}(t) \equiv \mathbf{c}_{n}=1$ and $n=N=\operatorname{deg} P$. Such equations were studied by many authors (see, for example, $[9,10,13,14,17,18,20]$ and some others).

Example 6.7. Let us consider the complex equation

$$
\dot{u}=u^{n}+c(t),
$$

where $c(t) \in C_{+}^{0}(T)$ for given period $T>0$ and integer $n \geqslant 2$. Remark then for $n=2$ it is the Riccati equation.

Apply the main Theorem 2.4. We need to verify the assumption (v). Let next $\mathbf{c} \in \mathbb{C}$ is a mean value of $c(t)$. Then, the associated polynomial $P(z)=z^{n}+\mathbf{c}$. If $\mathbf{c} \neq 0$, then it has $n$ distinct complex roots $z_{1}, \ldots, z_{n}$ and

$$
r_{0}=\left|z_{i}\right|=|\mathbf{c}|^{1 / n}
$$

If $\mathbf{c}=0$, then the associated polynomial $P(z)=z^{n}$ has only one root $z_{0}=0$. The functions $\omega(x)$ and $l\left(x, r_{0}\right)$ defined by formulae

$$
\omega(x)=x^{n}+\|c\|_{0}, \quad l\left(x, r_{0}\right)=\frac{2}{T}\left(x-r_{0}\right)
$$

For the verification of the assumption (v) we will use Property 2.5. Find out the root $r>0$ of the equation $\omega^{\prime}(r)=2 / T$. We get

$$
r=\left(\frac{2}{n T}\right)^{1 /(n-1)} .
$$

The inequality $\left\|c_{1}\right\|_{0}<2 / T$ is fulfilled because $\left\|c_{1}\right\|_{0}=0$. The inequality $\omega(r)<l\left(r, r_{0}\right)$ in the case of (6.14) has a following form:

$$
r^{n}+\|c\|_{0}<\frac{2}{T}\left(r-r_{0}\right)
$$

After some calculations, we get a following theorem.

Property 6.8. Consider (6.14) with the coefficient $c(t) \in C_{+}^{0}(T)$ for given period $T>0$ and integer $n \geqslant 2$. If $\mathbf{c} \neq 0$ and the following inequality holds

$$
\|c\|_{0}+\frac{2}{T}|\mathbf{c}|^{1 / n}<(n-1)\left(\frac{2}{n T}\right)^{n /(n-1)},
$$


then (6.14) has at least $n$ distinct solutions in the algebra $C_{+}^{1}(T)$ with mean values $z_{1}, \ldots, z_{n}$ and $C^{0}$-norm less than $r$. If $\mathbf{c}=0$ and

$$
\|c\|_{0}<(n-1)\left(\frac{2}{n T}\right)^{n /(n-1)}
$$

then (6.14) has at least one solution in the algebra $C_{+}^{1}(T)$ with mean value 0 and $C^{0}$-norm less than $r$.

Example 6.9. Let us consider (6.14) for $n=2$ (Riccati equation)

$$
\dot{u}(t)=u^{2}(t)+c(t),
$$

where the coefficient

$$
c(t)=\operatorname{Re}^{i v t} \pm r_{0}^{2}, \quad v=\frac{2 \pi}{T},
$$

belongs to the algebra $C_{+}^{0}(T)$ for given period $T>0$. Assume that real $R, r_{0}>0$. Then, $\|c(t)\|_{0}=R$ and $P(z)=z^{2} \pm r_{0}^{2}$.

Remark 6.10. If $n=2$, then $r=1 / T$ and the inequality (6.19) has a very simple form

$$
R+\frac{2}{T} r_{0}<\frac{1}{T^{2}}
$$

The inequality (6.23) is a sufficient condition for existence of two distinct solutions in the Banach algebra $C_{+}^{1}(T)$ of $(6.21)$ with $C^{0}$-norm less than $r=1 / T$.

Remark that all examples of (6.14) with periodic $c(t)$ but without any periodic solution as these given by Lloyd [13], Hassan [12], and Campos and Ortega [10] have to be constructed with $c(t)$ being a function outside the algebra $C_{+}^{0}$. Indeed in all the mentioned examples $c(t)$ is a real periodic function, that is, $c: \mathbb{R} \rightarrow \mathbb{R} \subset \mathbb{C}$. On the other hand we have a direct observation.

Remark 6.11. Suppose that a function $u(t) \in C_{+}^{0}(T)$ is a real-valued. Then $u(t)$ is a constant function.

Example 6.12. In [20], by use of the Fourier series, Miklaszewski showed (under a conjecture) that there exists $R=R_{0}>0$ such that the Riccati equation

$$
\dot{u}(t)=u^{2}(t)+R \mathbf{e}^{i t}
$$

has not $2 \pi$-periodic solution. Here $c(t)=\operatorname{Re}^{i t}$ belongs to $C_{+}^{0}(2 \pi)$ but $\mathbf{c}=0$. Write the inequality (6.23) for $T=2 \pi$ and $r_{0}=0$. We get

$$
R<\frac{1}{4 \pi^{2}}
$$

a sufficient condition for an existence of the solution $u(t) \in C_{+}^{1}(2 \pi)$ of (6.24) with

$$
\mathbf{A}(u)=0, \quad\|u\|_{0} \leqslant \frac{1}{2 \pi}-\sqrt{\frac{1}{4 \pi^{2}}-R .}
$$




\section{Acknowledgment}

The Research was supported by Poland Grant KBN no. 2 PO3A 04522.

\section{References}

[1] J. Andres, A nontrivial example of application of the Nielsen fixed-point theory to differential systems: problem of Jean Leray, Proceedings of the American Mathematical Society 128 (2000), no. 10, 2921-2931.

[2] J. Andres, L. Górniewicz, and J. Jezierski, A generalized Nielsen number and multiplicity results for differential inclusions, Topology and its Applications 100 (2000), no. 2-3, 193-209.

[3] A. Borisovich, Z. Kucharski, and W. Marzantowicz, Nielsen numbers and lower estimates for the number of solutions to a certain system of nonlinear integral equations, Applied Aspects of Global Analysis, Novoe Global. Anal., vol. 14, Voronezh University Press, Voronezh, 1994, pp. 3-10, 99.

[4] Some applications of the Nielsen number to algebraic sets, Proceedings of the Conference “Topological Methods in Nonlinear Analysis", December 1995, Gdańsk Scientific Society Press, Gdańsk, 1997, pp. 78-90.

[5] _ A multiplicity result for a system of real integral equations by use of the Nielsen number, Nielsen Theory and Reidemeister Torsion (Warsaw, 1996), Banach Center Publ., vol. 49, Polish Academy of Sciences, Warsaw, 1999, pp. 9-18.

[6] A. Borisovich and W. Marzantowicz, Multiplicity of periodic solutions for the planar polynomial equation, Nonlinear Analysis. Theory, Methods \& Applications. An International Multidisciplinary Journal. Series A: Theory and Methods 43 (2001), no. 2, 217-231.

[7] R. F. Brown, Retraction methods in Nielsen fixed point theory, Pacific Journal of Mathematics 115 (1984), no. 2, 277-297.

[8] _ Topological identification of multiple solutions to parametrized nonlinear equations, Pacific Journal of Mathematics 131 (1988), no. 1, 51-69.

[9] J. Campos, Möbius transformations and periodic solutions of complex Riccati equations, The Bulletin of the London Mathematical Society 29 (1997), no. 2, 205-215.

[10] J. Campos and R. Ortega, Nonexistence of periodic solutions of a complex Riccati equation, Differential and Integral Equations. An International Journal for Theory \& Applications 9 (1996), no. 2, 247-249.

[11] M. Fečkan, Nielsen fixed point theory and nonlinear equations, Journal of Differential Equations 106 (1993), no. 2, 312-331.

[12] H. S. Hassan, On the set of periodic solutions of differential equations of Riccati type, Proceedings of the Edinburgh Mathematical Society. Series II 27 (1984), no. 2, 195-208.

[13] N. G. Lloyd, The number of periodic solutions of the equation $\dot{z}=z^{N}+p_{1}(t) z^{N-1}+\cdots+p_{N}(t)$, Proceedings of the London Mathematical Society. Third Series 27 (1973), 667-700.

[14] _ On a class of differential equations of Riccati type, Journal of the London Mathematical Society. Second Series 10 (1975), 1-10.

[15] R. Manásevich, J. Mawhin, and F. Zanolin, Hölder inequality and periodic solutions of some planar polynomial differential equations with periodic coefficients, Inequalities and Applications, World Sci. Ser. Appl. Anal., vol. 3, World Scientific, New Jersey, 1994, pp. 459-466.

[16] W. Marzantowicz, Periodic solutions of nonlinear problems with positive oriented periodic coefficients, Variational and Topological Methods in the Study of Nonlinear Phenomena (Pisa, 2000), Progr. Nonlinear Differential Equations Appl., vol. 49, Birkhäuser Boston, Massachusetts, 2002, pp. 43-63.

[17] J. Mawhin, Periodic solutions of some planar nonautonomous polynomial differential equations, Differential and Integral Equations. An International Journal for Theory and Applications 7 (1994), no. 3-4, 1055-1061. 
[18] Continuation theorems and periodic solutions of ordinary differential equations, Topological Methods in Differential Equations and Inclusions (Montreal, PQ, 1994), NATO Adv. Sci. Inst. Ser. C Math. Phys. Sci., vol. 472, Kluwer Academic, Dordrecht, 1995, pp. 291-375.

[19] J. Mawhin and M. Willem, Critical Point Theory and Hamiltonian Systems, Applied Mathematical Sciences, vol. 74, Springer, New York, 1989.

[20] D. Miklaszewski, An equation $\dot{z}=z^{2}+p(t)$ with no $2 \pi$-periodic solutions, Bulletin of the Belgian Mathematical Society. Simon Stevin 3 (1996), no. 2, 239-242.

[21] R. Srzednicki, On periodic solutions of planar polynomial differential equations with periodic coefficients, Journal of Differential Equations 114 (1994), no. 1, 77-100.

[22] H. Żoładek, The method of holomorphic foliations in planar periodic systems: the case of Riccati equations, Journal of Differential Equations 165 (2000), no. 1, 143-173.

Andrei Borisovich: Institute of Mathematics, University of Gdańsk, ul. Wita Stwosza 57, 80-952 Gdańsk, Poland

E-mail address: andbor@math.univ.gda.pl

Wacław Marzantowicz: Faculty of Mathematics and Computer Science, Adam Mickiewicz University of Poznań, ul. Umultowska 87, 61-614 Poznań, Poland E-mail address: marzan@math.amu.edu.pl 